\title{
Sentiment Analysis of Political Reviews in Punjabi Language
}

\author{
Parul Arora \\ M.Tech Scholar \\ Department of Computer Engineering, Punjabi \\ University, Patiala
}

\author{
Brahmaleen Kaur \\ Assistant Professor, \\ Department of Computer Engineering, \\ Punjabi University, Patiala
}

\begin{abstract}
Sentiment Analysis is to distinguish and group the assessments/feelings/opinions in composed content. Till date, English Language incorporates the majority of the examination work around there. In this paper, we talked about the different methodologies used to finish the opinion investigation and exploration work accomplished for Indian Languages like Hindi, Bengali and Telugu. An approach is proposed to determine the sentiment orientation i.e. polarity of the Punjabi reviews by scoring method. Sentiment analysis is needed to be performed in Punjabi language because of the increase in Punjabi data on the web. Separate positive and negative condensed results are created which is useful for the client in choice making. We contrasted the outcomes and right now existing methodologies.
\end{abstract}

\section{General Terms}

Sentiment Analysis, Punjabi,Political Reviews, Algorithms.

\section{Keywords}

Sentiment Analysis, Punjabi Language, Senti Word Net

\section{INTRODUCTION}

The time of Web 2.0 has brought about era of inconceivable measure of client created substance. The creating innovation without breaking a sweat of reach ability and better integration has lead to broad utilization of sites, discussions, e-news, and surveys channels and the long range interpersonal communication stages, for example, Face book, Twitter. This informal communication stages have exponentially expanded the measure of data produced on consistent schedule.

\section{SENTIMENT ANALYSIS}

Sentiment Analysis manages examining feelings, sentiments, and the mentality of a speaker or an author from a given bit of text. SA helps in deciding the elements and subject from text towards which slant is sentiment is determined. SubjectivityDemonstrates that the content contains/bears presumption content. Sentiment are ordered as objective (truths), positive (speaks to a condition of joy, bliss, delight or fulfillment) or negative (speaks to a condition of distress, misgiving, bitterness or dissatisfaction). On the basis of degree of polarity, a score can be given to the sentiment [2]. In this manner, there are two research tips; first classifying the polarity of text to express the feeling in positive, negative or neutral. A few samples:-This motion picture by Aamir Khan and Kajol is great. (This sentence has a sentiment; it discusses the motion picture and the essayist's emotions about same "superb" and consequently its subjective) Objectivity- Shows that the content is without sentiment content. Second is identification of subjectivity or objectivity [3,4]. Due to its many aspects it is often referred to with different names such as opinion mining, sentiment classification, sentiment analysis, and sentiment extraction [5]. The thought of other individuals give data in choice making procedure [6].Each individual shares his or her data in social network sites, online journals, blogs, product review websites and web-forums. From all social network sites like Twitter and Face book, etrade sites, blogs; surveys and opinions of clients can be found. All these surveys and conclusions help the individuals and business associations. These reviews and opinions additionally help the researchers that how to analyze and summarize the opinions expressed in this huge measure of content information. This motion picture stars Aamir Khan and Kajol. The subjective content can be further sorted into three general classifications focused around the slants expressed in the content. Positive- The movie was fabulous (English) ढिलम घणुउ माठटान मी (Punjabi), Negative- Movie was horrible. (English) दिलभ घगुउ किभातव मी (Punjabi)

\subsection{Literature Review}

RaviChandran.et.al [7] has worked on the problem of detecting polarity of words by and detected about polarity of a word to be either positive or negative. For example, words such as good, beautiful, and wonderful are considered as positive words; whereas words such as bad, ugly, and sad are considered negative words. We treat polarity detection as a semi-supervised label propagation problem in a graph. In the graph, each node represents a word whose polarity is to be determined. Each weighted edge encodes a relation that exists between two words. Each node (word) can have two labels: positive or negative. We study this framework in two different resource availability scenarios using Word Net and Open Office the saurus when Word Net is not available. We report our results on three different languages: English, French, and Hindi. Our results indicate that label propagation improves significantly over the baseline and other semi supervised learning methods like Min-cuts and Randomized Min-cuts for this task (2009).Singh et.al. [8] has stated in (2013) an experimental work on a new kind of domain specific featurebased heuristic for aspect-level sentiment analysis of movie reviews. We have devised an aspect oriented scheme that analyses the textual reviews of a movie and assign it a sentiment label on each aspect. The scores on each aspect from multiple reviews are then aggregated and a net sentiment profile of the movie is generated on all parameters. We have used a Senti WordNet based scheme with two different linguistic feature selections comprising of adjectives, adverbs and verbs and n-gram feature extraction. We have also used our Senti WordNet scheme to compute the document-level sentiment for each movie reviewed and compared the results with results obtained using Alchemy API. The sentiment profile of a movie is also compared with the document-level 
sentiment result. The results obtained show that our scheme produces a more accurate and focused sentiment profile than the simple document-level sentiment analysis. There are broadly three types of approaches for sentiment classification of texts:(a) Using machine learning based text classifier -such as Naïve Bayes, SVM or KNN- with suitable feature selection scheme;(b) Using the unsupervised semantic orientation scheme of extracting relevant $n$-grams of the text and then labeling them either as positive or negative and consequentially the document; and(c) Using the Senti Word Net based publicly available library that provides positive, negative and neutral scores for words. Some of the relevant past works on sentiment classification can be found in [9](2003) , [10](2002), [11] (2005). Arora [11] has defined (2015) In this paper, Sentiment analysis is needed to be performed in Punjabi language. In this study will consist of algorithm, which has not been implemented yet but proposed. The Punjabi database (Word Net) has also not been used in the existing works. In future, we can try to come up for the Punjabi language, which does not utilize a have, word net but a proposed algorithm. The same will be implemented to translate English to Punjabi language. It can be easily used and applied to learn from the text more effectively.

\subsection{Present Work}

Punjabi Sentiment is a recently developed sentiment analysis for Punjabi language. It is an offline application developed in an ASP.NET language with SQL Server 2008. The main features of Punjabi Sentiment make an application that maintains the directory of positive, negative words, easy to operate, Using survey approach through which we find out the word is positive, negative, differentiate the words from the whole paragraph whether it is positive, negative. Differentiate the positive, negative with scores fetches from database from a whole sentence, Find out the total positive score and total negative score words in a sentence, better efficiency, dynamic based approach used in an algorithm.

\subsubsection{Working}

1. We develop a page for introducing the newly developed Punjabi Sentiment Analysis

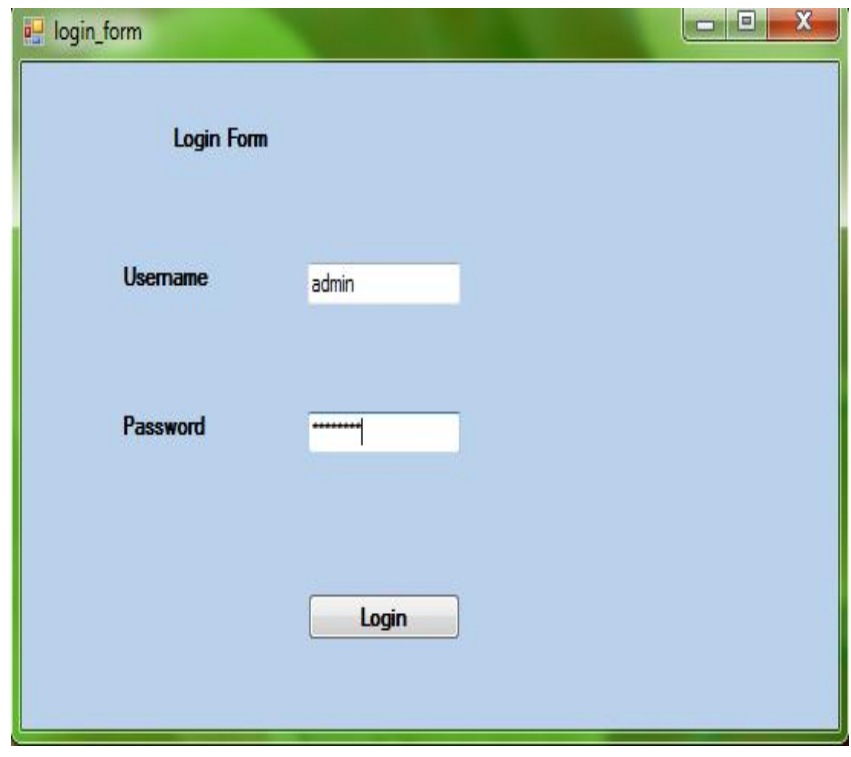

Figure 1.1Snapshot of login form page of Punjabi Sentiment Analysis

2. It describes the working of sentiment analysis

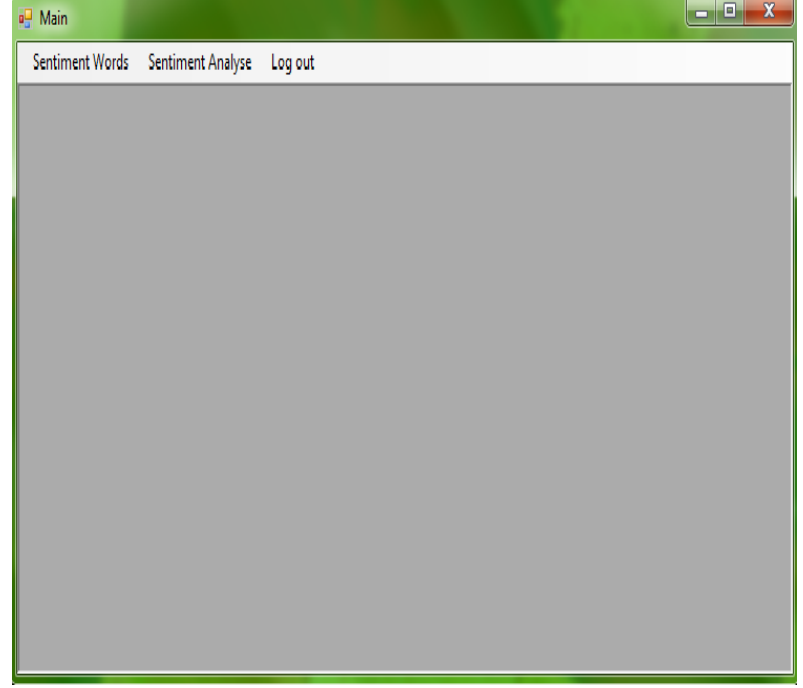

Figure 1.2: Snapshot of working of sentiment analysis

3. The user is add new word. He enters the word id by filling Punjabi word is either positive or negative and give with score value to a particular and save the word in the database as show below

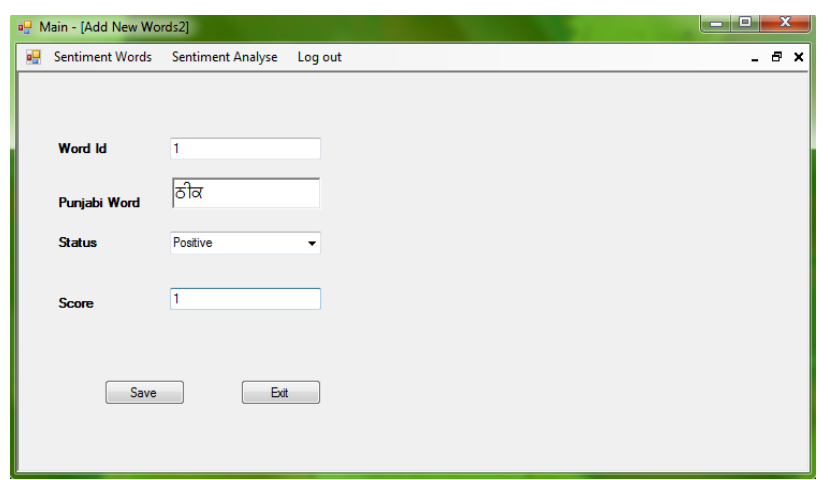

Figure 1.3: Snapshot to store Punjabi word

4. It depicts the data is entered by user for view words having fields of word id, Punjabi words, status and score as shown in the figure below.

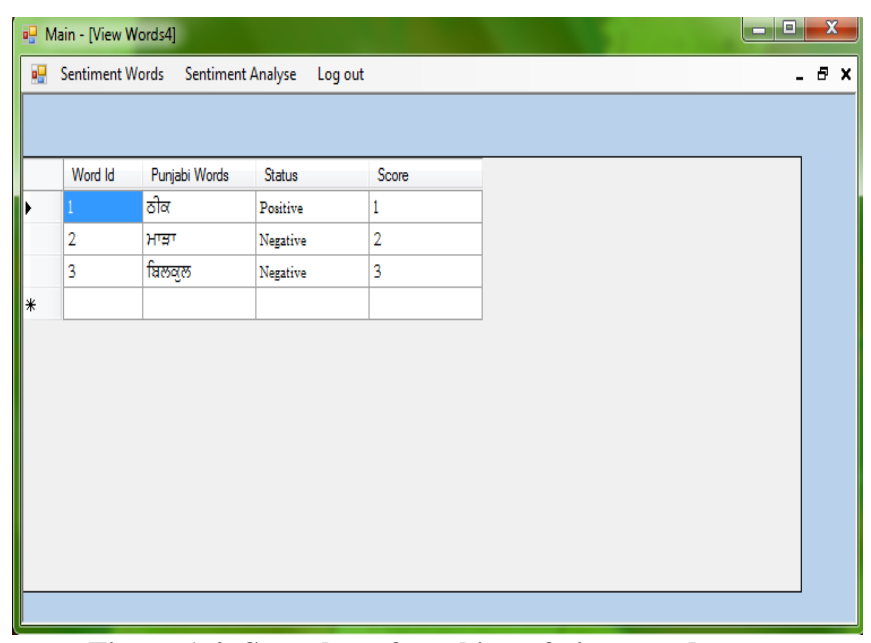

Figure 1.4: Snapshot of working of view words

5. When user enters a paragraph. Its automatically differentiate the sentence with positive sentence (green 
color) and negative sentence (red color), by clicking on result button as shown in the figure

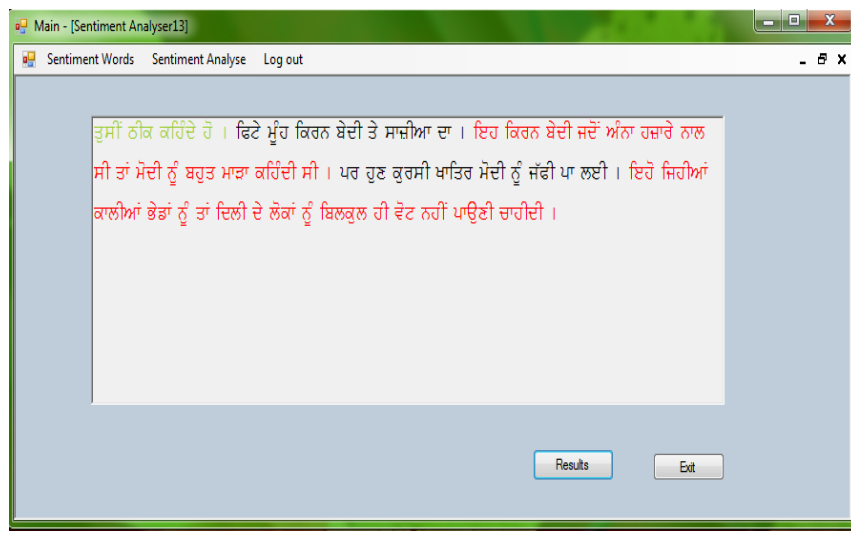

Figure 1.5: Snapshot working of sentiment analyze

6. Now shows the view result in a sentence with total score, status and count words in a sentence

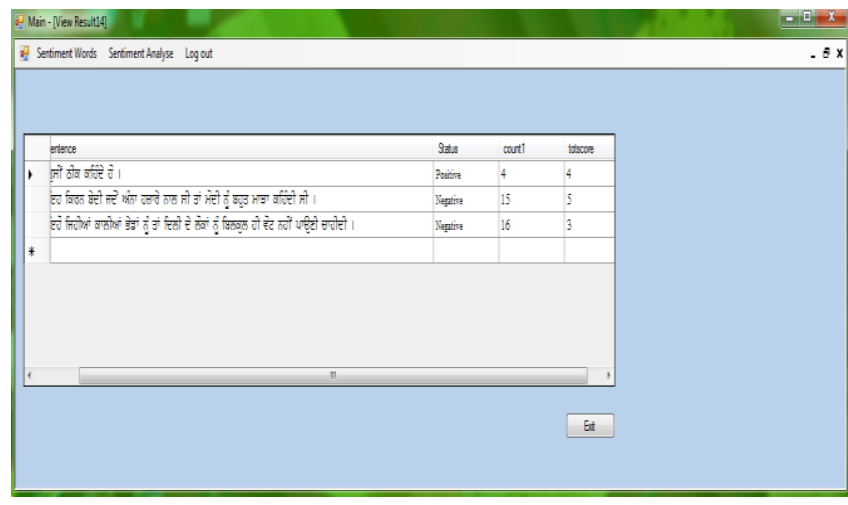

Figure 1.6: Snapshot of view result in sentiment analyze

7. It shows the view result with number of words and total number of words in a sentence.

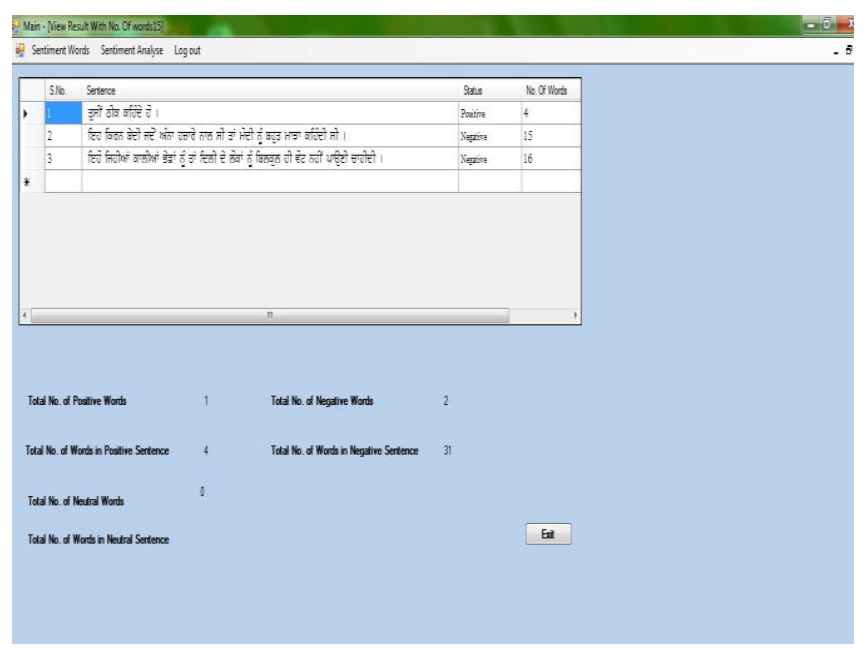

Figure 1.7: Snapshot of view result in sentiment analyze

8. In this view result with graph in a sentence is represented.

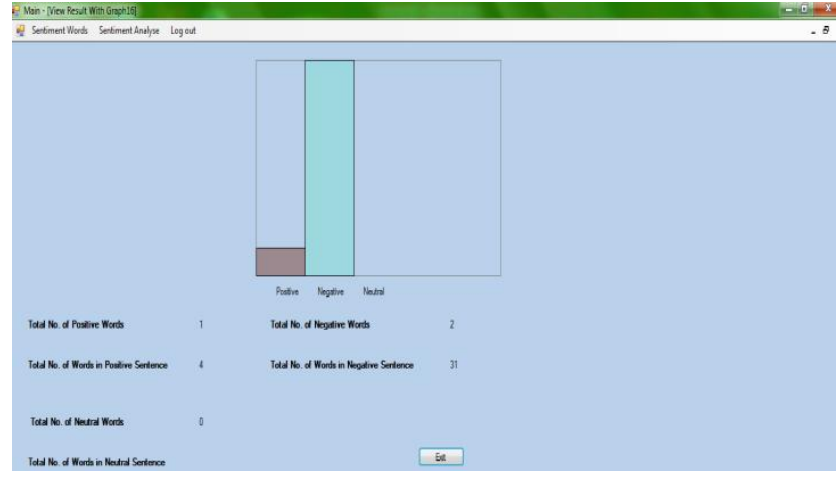

Figure 1.8: Snapshot of view result with graph

\section{PARAMETERS}

1. 1 Find out the status of the given result.

2. Sense of sentences

3. Using survey approach through which we find out the word is positive, negative.

4. Differentiate the words from the whole paragraph whether it is positive, negative.

5. Differentiate the positive, negative with scores fetches from database from a whole sentence.

6. Find out the total positive score and total negative score words in a sentence.

7. An Algorithm is made and simple scoring method which provides the better efficiency.

8. A dynamic based approach used in an algorithm.

\subsection{ALGORITHM}

Step 1: Collect the reviews in a paragraph.

Step 2: Store all words in a string array paragraph.

Step 3: Initialize the flag status as POSITIVE.

Step 4: The selected word from the paragraph.

Step 5: If the result is POSITIVE.

(a)Change the flag status as TRUE

End of Step 4 Loop

Step 6: If the flag status is TRUE.

(a) The each letter of the paragraph word with one by one letter of all the words of the database.

Step 7: If the result is TRUE.

Step 8: Show the sentence is positive than highlight the whole sentence positive from a paragraph.

End of Step 5 if statement.

End of Step 6 if statement.

Step9: Either the sentence is negative than highlight the whole sentence from a paragraph and count the number of words.

Step10: The result is shown in graph form and show the total scores of a word.

\section{RESULTS AND DISCUSSIONS}

The concept of opinion in the context of sentiment analysis the main task of sentiment analysis is to find out the positive, 
negative words from selected paragraphs. Existing studies about them have mostly focused on their intersections with opinion. However, we should realize that all these concepts and their definitions are well defined. Here are some results that have been used for evaluation of this study :- Formula used to show the output:-

Percentage for positive words: $\%$ of positive words $=$ Total positive words $* 100 /$ Number of positive words in a paragraph.

Percentage for negative words : $\%$ of negative words $=$ Total negative words $* 100 /$ Number of negative words in a paragraph

Find out the score

Set positive score $=0$

Positive score $=$ positive score + Fetched score from identified positive word.

\section{ACKNOWLEDGMENTS}

I am thankful to my guide Brahmaleen Kaur, Assistant Professor in Department of Computer Science Engineering for their valuable support. Also I am thankful to my Department for the technical support.

\section{FUTURE SCOPE}

In India, there are 22 official languages and 13 languages have more than 10 million speakers. With multiple sources of data available for each language, it is easy to gather data and analyze them. In context to Indian Languages, earlier work done for sentiment analysis has been on Bengali and Hindi, rest all the languages are unexplored .Through a deep study of existing research papers it has been found that one of the unexplored languages is Punjabi. The Punjabi database (Word Net) has also not been used in the existing works. The current version of this algorithm does not perform Word Sense Disambiguation (WSD).

\section{REFERENCES}

[1] Deepak Ravichandran," Semi-Supervised Polarity Lexicon Induction", Proceedings of the Third IEEE International Conference on Data Mining, 2009.

[2] V.K. Singh, R. Piryani," Sentiment Analysis of Movie Reviews A new Feature-based Heuristic for Aspect-level Sentiment Classification”, IEEE, 2013.
[3] Er.Parul Arora, Er.Brahmaleen Kaur, "An approach for Sentiment Analysis for Punjabi Text. International Journal of Information Technology\& Computer Sciences Perspectives $($ Pezzottaite Journals. 1452|Page, Volume 4, Number 2, April - June 2015 ISSN (Print):2319 9016, (Online):2319-9024 PEZZOTTAITE JOURNALS SJIF (2012): 3.201, SJIF (2013): 5.058, SJIF (2014): 5.891

[4] K. Dave, S. Lawerence \& D. Pennock, "Mining the Peanut Gallery-Opinion Extraction and Semantic Classification of Product Reviews", Proceedings of the 12th International World Wide Web Conference, pp. 519-528, 2003.

[5] P. Turney, "Thumbs up or thumbs down? Semantic orientation applied to unsupervised classification of reviews", Proceedings of ACL-02, 40th Annual Meeting of the Association for Computational Linguistics, pp. 417-424, Philadelphia, US, 2002.

[6] A. Esuli \& F.Sebastiani, "Determining the Semantic Orientation of terms through gloss analysis", Proceedings of CIKM-05, $14^{\text {th }}$ ACM International Conference on Information and Knowledge Management, pp. 617-624, Bremen, DE, 2005.

[7] Christos Troussas," Predicting Movie Sales Revenue using Online Reviews"IJRRC,2012.

[8] Troussas, "Sentiment analysis of Face book statuses using Naive Bayes classifier for language learning",ACM International Conference on Information and Knowledge Management, 2013.

[9] Ms.K.Mouthami, Arzu Baloglu "Sentiment Analysis and Classification Based On Textual Reviews",Fifth International Conference on Internet and Web Applications and Services, 2010

[10] Neethu M S, Christos TroussasA. Agarwal, B. Xie, I. Vovsha, O. Rambow, R. Passonneau, "Sentiment Analysis in Twitter using Machine Learning Techniques",Sentiment analysis of Twitter data", LSM '11 Proceedings of the Workshop on Languages in Social Media, Association for Computational Linguistics, pp. 30-38, 2011. 